\title{
THE GLOBAL DIMENSION THEOREM FOR WEIGHTED CONVOLUTION ALGEBRAS
}

\author{
by F. GHAHRAMANI ${ }^{1}$ and YU. V. SELIVANOV ${ }^{2}$
}

(Received 27th August 1996)

\begin{abstract}
We show that the global dimension, $\operatorname{dg} A$, of every commutative Banach algebra $A$ whose radical is a weighted convolution algebra is strictly greater than one. As an application, we see that in this case $\mathcal{H}^{2}(A, X) \neq 0$ for some Banach $A$-bimodule $X$ and thus there exists an unsplittable singular extension of the algebra $A$.
\end{abstract}

1995 Mathematics subject classification: 46J99, 46M20, 18 G20.

\section{Introduction}

This paper is concerned with a problem on "forbidden values" for homological dimensions of commutative Banach algebras. The existence of "forbidden values" for certain homological characteristics of Banach algebras is a well-known phenomenon that has no analogue in abstract algebra. This phenomenon is primarily associated with the existence of non-complemented closed subspaces and is also related to certain other geometric properties of Banach spaces.

We recall that the "global dimension theorem" of Helemskii [11] (see also [12]) states that every commutative Banach algebra with infinite spectrum has global homological dimension strictly greater than one.

How essential is the assumption that the spectrum is infinite in the result of Helemskii? Is the global dimension always strictly greater than one in the class of commutative Banach algebras? Of commutative radical Banach algebras?

These questions were raised in [13] and [14]. No complete answers to these questions are known.

In addition to commutative Banach algebras with infinite spectra, the inequality in question has also been proved for those commutative Banach algebras in which the multiplier seminorm is not equivalent to the original norm (see [17, Corollary 4]). Also this inequality has been proved for commutative Banach algebras without bounded approximate identities, provided that they have the bounded approximation property

\footnotetext{
${ }^{1}$ Research supported by NSERC grant OGP 003664.

${ }^{2}$ Partially supported by the Russian Foundation for Fundamental Research (grant 96-01-01036). Part of this research was carried out while the second author was a visitor of the University of Manitoba and then also at the Workshop on Complete Boundedness and Cohomology of Operator Algebras (Newcastle 1995).
} 
(see [18]). The result is also true for the Volterra algebra $L^{1}[0,1]$ (see [18]), which is a radical commutative Banach algebra with a bounded approximate identity.

In this paper we give a positive answer to the second and the third questions mentioned above, for Banach algebras whose radicals satisfy certain conditions. In particular, we show that, if $A$ is a commutative Banach algebra and its radical is a weighted convolution algebra on the half-line, then the global dimension of $A$ is strictly greater than one. As an application, we see that in this case $\mathcal{H}^{2}(A, X) \neq 0$ for some Banach $A$-bimodule $X$ and thus there exists an unsplittable singular extension of this algebra.

\section{Preliminaries}

Let $A$ be a commutative Banach algebra, not necessarily with an identity, and let $A_{+}$be its unitization. The categories of left Banach $A$-modules and Banach $A$ bimodules will be denoted respectively by $A$-mod and $A$-mod- $A$. If $X, Y \in A$-mod (resp. $A$-mod- $A$ ) then the corresponding sets of morphisms from $X$ to $Y$ will be denoted by ${ }_{A} h(X, Y)$ (resp. ${ }_{A} h_{A}(X, Y)$ ). Instead of ${ }_{A} h(X, X)$ we will write ${ }_{A} h(X)$. The Banach analogues of the notions of homological dimension of a module and of an algebra, which are the subject of our main theorems were introduced by Helemskii [10]. This theory is set out in detail in [13].

We recall that there is the so-called canonical morphism $\pi_{+}: A_{+} \hat{\otimes} X \rightarrow X$ associated with any $X \in A$-mod; this morphism is defined by $\pi_{+}(a \otimes x)=a \cdot x\left(a \in A_{+}, x \in X\right)$. Here $\hat{\otimes}$ denotes the projective tensor product (see [9]). The space $A_{+} \hat{\otimes} X$ is a left Banach $A$-module with the outer multiplication, well-defined by $a \cdot(b \otimes x)=$ $a b \otimes x\left(a \in A, b \in A_{+}, x \in X\right)$.

Recall that a left Banach $A$-module $X$ is projective if and only if the canonical morphism $\pi_{+}$has a right inverse morphism $\rho$; in this case $\rho$ is said to be a coretraction of the projective A-module $X$.

We now recall that a sequence of left Banach $A$-modules and morphisms

$$
0 \stackrel{d_{-1}}{\longleftarrow} X_{0} \stackrel{d_{0}}{\longleftarrow} X_{1} \stackrel{d_{1}}{\longleftarrow} X_{2} \stackrel{d_{2}}{\longleftarrow} \cdots(X)
$$

is said to be a (chain) complex in $A$-mod, if the composition of any two consecutive morphisms is zero. The complex is said to be exact if $\operatorname{Ker} d_{n-1}=\operatorname{Im} d_{n}$ for all $n$, and admissible if it splits as a complex of Banach spaces. So an admissible complex is, in particular, exact. For $X \in A$-mod, a complex $\mathfrak{X}$ in $A$-mod together with a morphism $e \in \in_{A} h\left(X_{0}, X\right)$ is called a resolution of $X$ if

$$
0 \longleftarrow X \stackrel{e}{\longleftarrow} X_{0} \stackrel{d_{0}}{\longleftarrow} X_{1} \stackrel{d_{1}}{\longleftarrow} \cdots
$$

is an admissible complex. The resolution is said to be projective, if all the modules in $\mathfrak{X}$ are projective.

The length of the resolution $(\mathfrak{X}, e)$ is the smallest $n$ such that $X_{k}=0$ for $k>n$, or 
$\infty$ if there is no such $n$. The homological dimension of $X \in A$-mod, denoted by $\mathrm{dh}_{A} X$, is the length of the shortest projective resolution of $X$, while the global (homological) dimension of the algebra $A, \operatorname{dg} A$, is the upper bound of the values $\operatorname{dh}_{A} X$ taken over all $X \in A$-mod. Thus the "global dimension theorem" of Helemskii, after appropriate "unmasking", reads as follows: if $A$ is a commutative Banach algebra with infinite spectrum, then there is a left Banach $A$-module $X$ such that there is no resolution

$$
0 \longleftarrow X \stackrel{e}{\longleftarrow} X_{0} \stackrel{d_{0}}{\longleftarrow} X_{1} \longleftarrow 0
$$

with $X_{0}$ and $X_{1}$ projective.

We will introduce other terminology and notation as the need arises.

\section{Reducing the problem}

In this section we shall prove a number of properties for a commutative Banach algebra $A$ with $\operatorname{dg} A \leq 1$. The first property is widely known, we give a proof for completeness.

Lemma 2.1. Let $A$ be a commutative Banach algebra with $\operatorname{dg} A \leq 1$, and let $I$ be a complemented (that is, having Banach complement) closed ideal in $A$. Then I is projective in A-mod.

Proof. The condition implies that the short exact sequence

$$
0 \leftarrow A_{+} / I \leftarrow A_{+} \leftarrow I \leftarrow 0
$$

is a resolution of the $A$-module $A_{+} / I$. Since $\mathrm{dh}_{A} A_{+} / I \leq 1$, the assertion follows from [13, Theorem III. 5.4].

For Banach spaces $E$ and $F$, let $\mathfrak{B}(E, F)$ be the Banach space of all continuous linear operators from $E$ to $F$. We write $\mathfrak{B}(E)$ for $\mathfrak{B}(E, E)$. For the definitions of the approximation property (AP for short) and the bounded approximation property (BAP for short) for Banach spaces, see for example [16]. These properties are discussed also in [9], [8], [13] and [20].

Lemma 2.2. (see [19, Theorem 1]). Let $A$ be a commutative Banach algebra, let $X$ be a projective left Banach $A$-module, and let $\rho$ be a coretraction of $X$. Then the formula $P(\varphi)=\pi_{+}\left(1_{A_{+}} \hat{\otimes} \varphi\right) \rho$ defines a continuous linear operator $P: \mathfrak{B}(X) \rightarrow{ }_{A} h(X)$ such that:

(i) $P(\varphi)=\varphi$ for all $\varphi \in_{A} h(X)$;

(ii) the image $P(\varphi)$ of each finite-rank operator $\varphi \in \mathfrak{B}(X)$ has a representation of the form $P(\varphi) x=\sum_{i=1}^{n} T_{i}(x) \cdot y_{i}(x \in X)$, where $T_{i} \in_{A} h\left(X, A_{+}\right), y_{i} \in X(1 \leq i \leq n)$; 
(iii) if $X$ has $A P$, then the identity operator $1_{X}$ is the uniform limit over the compact subsets of $X$ of operators of the form $P(\varphi)$, where $\varphi \in \mathfrak{B}(X)$ is a finite-rank operator;

(iv) if $X$ has $B A P$, then there exists a bounded net of finite-rank operators $\left\{\varphi_{\lambda}\right\} \subset \mathfrak{B}(X)$ such that $P\left(\varphi_{\lambda}\right) x \rightarrow x$ for each $x \in X$.

We recall that in a commutative Bànach algebra $A$, an approximate identity $\left\{e_{\lambda}: \lambda \in \wedge\right\}$ is said to be operator-bounded if there is some constant $C$ such that

$$
\left\|a e_{\lambda}\right\| \leq C\|a\|(a \in A, \lambda \in \wedge) .
$$

We denote by $\overline{A^{2}}$ the closure of the linear span of elements of the form $a b(a, b \in A)$. A Banach algebra $A$ is said to be idempotent if $\overline{A^{2}}=A$.

The following theorem is related to [18, Theorem 1].

Theorem 2.3. Let A be a commutative Banach algebra, let I be a projective closed ideal in $A$ and suppose that $T(I) \subset I$ for each $T \epsilon_{A} h\left(I, A_{+}\right)$. Then:

(i) if I has $A P$, then I is idempotent and possesses an approximate identity;

(ii) if I has BAP, then I possesses an operator-bounded approximate identity.

Proof. (i) Let us assume that $I$ has AP. We shall prove that for each compact set $K \subset I$ and any neighbourhood $U$ of zero in $I$ there exists an element $e \in I$ such that $a-a e \in U$ for all $a \in K$. Indeed, by Lemma 2.2, the identity operator $1_{I}$ can be approximated uniformly on $K$ by operators of the form $P(\varphi)$, where $\varphi \in \mathfrak{B}(I)$ is a finite-rank operator; in this case

$$
P(\varphi) a=\sum_{i=1}^{n} T_{i}(a) b_{i}(a \in I)
$$

where $T_{i} \in_{A} h\left(I, A_{+}\right), b_{i} \in I(1 \leq i \leq n)$. Since $T(I) \subset I$ for any $T \in_{A} h\left(I, A_{+}\right)$, we see that

$$
P(\varphi) a=\sum_{i=1}^{n} T_{i}\left(b_{i} a\right)=a \sum_{i=1}^{n} T_{i}\left(b_{i}\right)=a e(a \in I),
$$

where $e=\sum_{i=1}^{n} T_{i}\left(b_{i}\right) \in I$. The rest is clear.

(ii) Now suppose that $I$ has BAP. By Lemma 2.2, for some constant $C_{1}>0$ and for any $a \in I$ we have $a=\lim _{\lambda} P\left(\varphi_{\lambda}\right) a$, where $\left\{\varphi_{\lambda}: \lambda \in \wedge\right\} \subset \mathfrak{B}(I)$ is a net of finite-rank operators with $\left\|\varphi_{\lambda}\right\| \leq C_{1}$ for all $\lambda \in \wedge$. Then, clearly, for all $\lambda$

$$
P\left(\varphi_{\lambda}\right) a=a e_{\lambda}(a \in I),
$$

where $e_{\lambda} \in I$, and 


$$
\left\|a e_{\lambda}\right\|=\left\|P\left(\varphi_{\lambda}\right) a\right\| \leq C_{2}\|a\|,
$$

where $C_{2}=C_{1}\|P\|$. Thus, $\left\{e_{\lambda}\right\}$ is an operator-bounded approximate identity in $I$.

From Lemma 2.1 and Theorem 2.3 we obtain the following.

Corollary 2.4. Let $A$ be a commutative Banach algebra with $\operatorname{dg} A \leq 1$, let I be a non-idempotent, complemented closed ideal in $A$ and suppose that $\overline{A \cdot I}=I$ and $I$ has $A P$. Then there exists a morphism $T \in_{A} h(I, A)$ such that $T(I) \notin I$.

Now we recall the basic facts on Banach modules of continuous linear multipliers and their homological properties.

Let us recall from [21] that, for a commutative Banach algebra $A$, a map $T: A \rightarrow A$ is a multiplier if $T(a b)=a T(b)$. Let $M(A)$ denote the set of all continuous linear multipliers on $A$ (that is, $M(A)={ }_{A} h(A)$ ). If $A$ is a commutative Banach algebra without non-zero annihilators (for example, if $A$ possesses an approximate identity), then each multiplier belongs to $M(A)$ (see [21]).

The set $M(A)$ is a closed subalgebra of the Banach algebra $\mathfrak{B}(A)$, and $M(A)$ is a left Banach $A$-module (see [17]) provided that the outer multiplication is defined by

$$
(a \cdot T)(b)=T(b a)(a, b \in A) .
$$

Since $M(A)$ contains the identity operator $1_{A}$, we can consider the morphism of left $A$-modules $\alpha: A_{+} \rightarrow M(A)$ given by

$$
\alpha(a)=a \cdot 1_{A}=T_{a},
$$

where $T_{a}(b)=b a(b \in A)$. The closure of the image of the morphism $\alpha$ is denoted by $M_{+}(A)$. Clearly $M_{+}(A)$ is a closed submodule of the left $A$-module $M(A)$.

Recall from [17] that the so-called multiplier seminorm $\|\cdot\|_{M}$ on a Banach algebra $A$ is

$$
\|a\|_{M}=\sup \{\|b a\|: b \in A,\|b\| \leq 1\} .
$$

Evidently $\|a\|_{M} \leq\|a\|(a \in A)$. Also it is clear that, if $A$ possesses a bounded approximate identity, then $\|\cdot\|$ and $\|\cdot\|_{M}$ are equivalent. In general the converse is false. However it will be true in the case when $A$ has an operator-bounded approximate identity.

Lemma 2.5. (see [17, Theorem 3]). Let $A$ be a commutative Banach algebra such that $\mathrm{dh}_{A} A=n$ is finite. Then $\mathrm{dh}_{A} M_{+}(A) \leq n+2$. If, in addition, $\|\cdot\|$ and $\|\cdot\|_{M}$ are not equivalent, then $\mathrm{dh}_{A} M_{+}(A)=n+2$.

Corollary 2.6. Let $A$ be a commutative Banach algebra with $\operatorname{dg} A \leq 1$. Then the multiplier seminorm on $A$ is equivalent to the original norm. 
The following corollary is a consequence of Lemma 2.1, Theorem 2.3 and Corollary 2.6 .

Corollary 2.7. Let $A$ be a commutative Banach algebra with $\operatorname{dg} A \leq 1$ and suppose that $A$ has $B A P$. Let $A$ satisfy at least one of the following conditions:

(i) $T(A) \subset A$ for each $T \in \in_{A} h\left(A, A_{+}\right)$;

(ii) $A$ is idempotent.

Then A possesses a bounded approximate identity.

Recall [13, Theorem IV.3.16] that the condition (ii) in Corollary 2.7 holds automatically, if $\infty$ belongs to the Shilov boundary of the spectrum of the algebra $A_{+}$ (for example, if this spectrum is finite).

Corollary 2.8. Let $A$ be a commutative Banach algebra with finite spectrum. Suppose that $\operatorname{dg} A \leq 1$ and $A$ has $B A P$. Then $A$ possesses a bounded approximate identity.

Recall from [13] that a left Banach module $Y$ over a Banach algebra $A$ is said to be flat if, for any admissible complex $\mathfrak{X}$ of right Banach $A$-modules, the complex $\mathfrak{X} \hat{\otimes}_{A} Y$ is exact.

Lemma 2.9. (see [13, Theorem VII.1.20]). Let $A$ be a commutative Banach algebra, and let $I$ be a complemented closed ideal in $A$. Then the following conditions are equivalent:

(i) I has a bounded approximate identity;

(ii) $A_{+} / I$ is a flat left Banach A-module.

Theorem 2.10. Let $A$ be a commutative Banach algebra with finite spectrum. Suppose that $\operatorname{dg} A \leq 1$ and $A$ has BAP. Then any finite-dimensional left Banach $A$-module is flat; and as a consequence, every closed ideal of finite codimension in $A$ has a bounded approximate identity.

Proof. We first assume that $X$ is a one-dimensional left Banach $A$-module. Clearly $X$ is isomorphic to the $A$-module $A_{+} / M$, where $M$ is a maximal ideal in $A_{+}$. Since, by [13, Corollary III.5.11],

$$
\operatorname{dg} M=\operatorname{dg} A_{+}=\operatorname{dg} A \leq 1,
$$

it follows from Corollary 2.8 that $M$ possesses a bounded approximate identity. Hence, by Lemma $2.9, X$ is flat.

Now consider the general case, where $X$ is a finite-dimensional left Banach $A$ module. Since $A$ is commutative, $X$ contains a chain of submodules 


$$
0=X_{0} \subset X_{1} \subset \ldots \subset X_{n-1} \subset X_{n}=X,
$$

such that every $X_{k} / X_{k-1}(1 \leq k \leq n)$ is one-dimensional. Since the complexes of left $A$ modules

$$
0 \leftarrow X_{k} / X_{k-1} \leftarrow X_{k} \leftarrow X_{k-1} \leftarrow 0
$$

are obviously admissible, Proposition [13, VII.1.17], applied first for the case $k=2$, and then with $k=3$, and so on, implies that $X$ is flat.

\section{Weighted convolution algebras}

Throughout this section we assume that $\omega$ is a radical weight function on $\mathbf{R}^{+}=[0, \infty)$; that is a positive submultiplicative measurable function on $\mathbf{R}^{+}$, with $\lim _{t \rightarrow \infty} \omega(t)^{1 / t}=0$. We let $L^{\prime}(\omega)$ be the Banach algebra of all equivalence classes of complex-valued functions, integrable with respect to $\omega d t$, where $d t$ is the Lebesgue measure, and the product is the convolution product

$$
(f * g)(x)=\int_{0}^{x} f(x-y) g(y) d y .
$$

Then $L^{\prime}(\omega)$ is a radical algebra. Throughout $\omega$ will be a radical weight such that $L^{1}(\omega)$ has a bounded approximate identity.

For a Radon measure $\mu$ (or a locally integrable function $f$ ) on $\mathbf{R}^{+}$we denote by $\alpha(\mu)$ (resp. $\alpha(f)$ ) the infimum of the support of $\mu$ (resp. $f$ ). For each $a>0$ we let $I_{a}=\left\{f \in L^{1}(\omega): \alpha(f) \geq a\right\}$. Then $I_{a}$ is a closed ideal of $L^{1}(\omega) ; I_{a}$ is called a standard ideal. Any closed ideal of $L^{1}(\omega)$ which is not of this form is called a non-standard ideal. As shown in [7], for many radical algebras $L^{\prime}(\omega)$, all the closed ideals in $L^{\prime}(\omega)$ are standard. In the other direction, H. G. Dales and J. P. McClure in [4] have given an example of a radical $L^{1}(\omega)$ with a non-standard closed ideal.

We let $M(\omega)$ be the space of all Radon measures $\mu$ on $\mathbf{R}^{+}$such that $\|\mu\|=\int_{\mathbf{R}^{+}} \omega(t) d|\mu|(t)<\infty$. Then with convolution product, $M(\omega)$ is a Banach algebra.

We recall Titchmarsh's convolution theorem: Suppose that $\mu$ and $v$ are non-zero Radon measures (or locally integrable functions) on $\mathbf{R}^{+}$. Then $\alpha(\mu * v)=\alpha(\mu)+\alpha(v)$. For a proof of this in the case of functions see [3, Theorem 3.10]. The extension to measures then follows from noticing that if $u(t) \equiv 1$, then $\alpha(\lambda * u)=\alpha(\lambda)$ for all Radon measures $\lambda$ on $\mathbf{R}^{+}$. It is easily verified that Titchmarsh's convolution theorem is equivalent to: $\mu * v=0$, if and only if $\mu=0$ or $v=0$. It is the latter version that we shall use.

The following lemma is a consequence of [1, Lemma 1.2].

Lemma 3.1. Let $\omega$ be a radical weight function. Then for each $a>0$

$$
\lim _{x \rightarrow \infty} \inf \omega(x+a) / \omega(x)=0 .
$$


Let $C_{0}(1 / \omega)$ be the space of all continuous functions $f$ on $\mathbf{R}^{+}$such that $\lim _{x \rightarrow \infty} \frac{f(x)}{\omega(x)}=0$. Then with the norm

$$
\|f\|=\sup \left\{\frac{|f(x)|}{\omega(x)}: x \in \mathbf{R}^{+}\right\}
$$

$C_{0}(1 / \omega)$ is a Banach space, whose dual can be identified with $M(\omega)$, via the pairing

$$
\langle\mu, f\rangle=\int_{\mathbf{R}^{+}} f(x) d \mu(x),\left(\mu \in M(\omega), f \in C_{0}(1 / \omega)\right) .
$$

In what follows, for each $a>0$, we let $J_{a}=\{\mu \in M(\omega): \alpha(\mu) \geq a\}$; then $J_{a}$ is obviously a closed ideal in $M(\omega)$, and $I_{a} \subset J_{a}$.

Lemma 3.2. Suppose that $T$ is a multiplier from $I_{a}$ into $L^{1}(\omega)$. Then:

(i) $T$ is continuous.

(ii) $T$ has an extension to a continuous multiplier from $J_{a}$ into $M(\omega)$.

Proof. By using the Closed Graph Theorem and Titchmarsh's convolution theorem we can easily prove (i). To prove (ii) we let $w k^{*}=\sigma\left[M(\omega), C_{0}(1 / \omega)\right]$, and we let $\left(e_{n}\right)$ be a bounded approximate identity of $L^{1}(\omega)$. First we show that $\left(T\left(\mu * e_{n}\right)\right)$ has a $w k^{*}$ limit in $M(\omega)$. Since it is bounded, by the Banach-Alaoglu theorem, it has a $w k^{*}$-cluster point. Suppose that for two subnets $\left(e_{n(i)}\right)$ and $\left(e_{n(j)}\right)$

$$
v=w k^{*}-\lim T\left(\mu * e_{n(i)}\right), \text { and } \eta=w k^{*}-\lim T\left(\mu * e_{n(j)}\right)
$$

Then for each $f \in I_{a}$, we have

$$
\begin{aligned}
& v * f=\left(w k^{*}-\lim T\left(\mu * e_{n(i)}\right)\right) * f=w k^{*}-\lim \left(T\left(\mu * e_{n(i)}\right) * f\right) \\
& =w k^{*}-\lim T\left(\mu * e_{n(i)} * f\right)=T(\mu * f)
\end{aligned}
$$

Similarly, $\eta * f=T(\mu * f)$, and so by Titchmarsh's convolution theorem we have, $v=\eta$, showing that the $w k^{*}-\lim T\left(\mu * e_{n}\right)$ exists in $M(\omega)$. Next we define $\bar{T}: J_{a} \rightarrow M(\omega)$, by $\bar{T}(\mu)=w k^{*}-\lim T\left(\mu * e_{n}\right)$. Then we proceed to prove that $\bar{T}$ is a multiplier. To this end, let $\mu_{1}, \mu_{2} \in J_{a}$ and $f \in I_{a}$. Then from equation (1) we have

$$
\begin{gathered}
\bar{T}\left(\mu_{1} * \mu_{2}\right) * f=T\left(\mu_{1} * \mu_{2} * f\right)= \\
\operatorname{norm}-\lim T\left(\mu_{1} * e_{n} * \mu_{2} * f\right)=\text { norm }-\lim T\left(\mu_{1} * e_{n}\right) * \mu_{2} * f=\bar{T}\left(\mu_{1}\right) * \mu_{2} * f .
\end{gathered}
$$

Hence

$$
\bar{T}\left(\mu_{1} * \mu_{2}\right)=\bar{T}\left(\mu_{1}\right) * \mu_{2},
$$

by Titchmarsh's convolution theorem, and the lemma is proved. 
Theorem 3.3. For each $a>0$, if $T$ is a multiplier from $I_{a}$ into $L^{\prime}(\omega)$, then $T$ maps into $I_{a}$.

Proof. Suppose $T: I_{a} \rightarrow L^{\prime}(\omega)$ is a multiplier. Let $\bar{T}: J_{a} \rightarrow M(\omega)$ be extension of $T$, as given in Lemma 3.2. Define $S: M(\omega) \rightarrow M(\omega)$, by $S(\mu)=\bar{T}\left(\mu * \delta_{a}\right)$, where $\delta_{a}$ is the point mass at $a$. We are going to show that $S$ is a multiplier of $M(\omega)$. For $\mu_{1}$, $\mu_{2} \in M(\omega)$ we have

$$
\begin{aligned}
S\left(\mu_{1} * \mu_{2}\right) * \delta_{a} & =\bar{T}\left(\mu_{1} * \mu_{2} * \delta_{a}\right) * \delta_{a}=\bar{T}\left(\mu_{1} * \delta_{a} * \mu_{2} * \delta_{a}\right) \\
& =\bar{T}\left(\mu_{1} * \delta_{a}\right) * \mu_{2} * \delta_{a}=S\left(\mu_{1}\right) * \mu_{2} * \delta_{a}
\end{aligned}
$$

Hence $S\left(\mu_{1} * \mu_{2}\right)=S\left(\mu_{1}\right) * \mu_{2}$, showing that $S$ is a multiplier. Since $M(\omega)$ is unital, there exists a measure $\mu \in M(\omega)$ such that for all $v \in M(\omega), S(v)=v * \mu$. Hence,

$$
\bar{T}\left(v * \delta_{a}\right)=v * \mu(v \in M(\omega)) \text {. }
$$

From equation (3), for any $x \in \mathbf{R}^{+}$and $v=\delta_{x}$, we have

$$
\bar{T}\left(\delta_{x+a}\right)=\delta_{x} * \mu .
$$

Then from $\frac{\left\|\bar{T}\left(\delta_{x+a}\right)\right\|}{\left\|\delta_{x+a}\right\|} \leq\|\bar{T}\|$, we obtain

$$
\frac{1}{\omega(x+a)} \int_{\mathbf{R}^{+}} \omega(x+y) d|\mu|(y) \leq\|\bar{T}\| .
$$

We use this to show that $\alpha(\mu) \geq a$.

Suppose, towards a contradiction, that $\alpha(\mu)<a$. Choose $b$ and $c$ such that $0<b<c<a$ and

$$
0<|\mu|([b, c])
$$

Set $\delta=\frac{1}{2}(a-c)$. Then since on each compact subset of $(0, \infty), \omega$ is bounded [15, Section 7.4] and $\omega$ is a radical weight we have

$$
K=\sup \{\omega(x): x \in[\delta, \infty)\}<\infty
$$

For every $x \in \mathbf{R}^{+}$, let $m(x)=\inf \{\omega(x+y): y \in[b, c]\}$. Then $m(x)>0$ [15, Section 7.4]. Hence for each $x$, there exists $z \in[b, c]$ such that

$$
\omega(x+z)<2 m(x) \text {. }
$$

From (4), (5) and (7) we have 


$$
\begin{aligned}
& \frac{\omega(x+z)}{\omega(x+a)}|\mu|([b, c]) \leq \frac{2 m(x)}{\omega(x+a)}|\mu|([b, c]) \\
& \leq \frac{2}{\omega(x+a)} \int_{[b, c]} \omega(x+y) d|\mu|(y) \leq 2\|\bar{T}\| .
\end{aligned}
$$

Hence for every $x \in \mathbf{R}^{+}$, there exists $z \in[b, c]$ with $M<\frac{\omega(x+a)}{\omega(x+z)}$, where $M=\mu([b, c]) /$ (2 $\|\bar{T}\|$ ). Then, by submultiplicativity of $\omega$ and using (6) we have

$$
\begin{gathered}
M<\frac{\omega(x+a)}{\omega(x+c+\delta)} \frac{\omega(x+c+\delta)}{\omega(x+z)} \\
\leq \frac{\omega(x+a)}{\omega(x+c+\delta)} \omega(c-z+\delta) \leq K \frac{\omega(x+a)}{\omega(x+c+\delta)} \quad\left(x \in \mathbf{R}^{+}\right) .
\end{gathered}
$$

By taking $\lim i n f_{x \rightarrow \infty}$ in (8) and using Lemma 3.1 a contradiction follows. Hence $\alpha(\mu) \geq a$. Now if $f \in I_{a}$ and $f$ has a compact support, then $f=g * \delta_{a}$, for some $g \in L^{1}(\omega)$. Then from (3) we have $T(f)=T\left(\delta_{a} * g\right)=g * \mu \in I_{a}$, since $\alpha(\mu) \geq a$. Since functions with compact support are dense in $I_{a}$, the proof is complete.

Remark 3.4. In, [5, Corollary 1.24] multipliers from $I_{a}$ into $I_{a}$ are characterized, when $\omega$ is continuous. That result combined with Theorem 3.3 yields:

Corollary 3.5. Suppose that $\omega$ is continuous, $L^{1}(\omega)$ is radical and has a bounded approximate identity. Then for each $a>0$, the following are equivalent:

(i) $T$ is a multiplier from $I_{a}$ into $L^{1}(\omega)$;

(ii) there exists a radon measure $\mu$ on $\mathbf{R}^{+}$such that $T(f)=f * \mu$, for all $f \in I_{a}$ and $\mu$ satisfies the growth condition:

$$
\sup \left\{\frac{1}{\omega(x)} \int_{\mathbf{R}^{+}} \omega(x+y) d|\mu|(y): x \in[a, \infty)\right\}<\infty
$$

(iii) $T$ is a multiplier from $I_{a}$ into $I_{a}$.

\section{The main results}

In this section we shall estimate the global dimension of commutative Banach algebras, assuming that their radicals have special properties. We shall apply these results to the study of cohomology groups and extensions of Banach algebras.

Theorem 4.1. Let $A$ be a commutative Banach algebra, and let $R$ be the radical of $A$. Suppose that $R$ has $B A P$ and does not possess a bounded approximate identity. Then $\operatorname{dg} A>1$. 
Proof. Because of the "global dimension theorem" of Helemskii (see [11]) we can assume that the spectrum of $A$ is finite. In this case $R$ is a closed ideal of finite codimension in $A$. Then clearly $R$ is a complemented closed subspace of $A$, and $A$ has BAP. Then Theorem 2.10 can be applied to complete the proof.

For example, let $R$ be the maximal ideal in the (local) Banach algebra $l^{\prime}(\omega)$, where $\omega$ is a radical weight (see [2]). It is obvious that $R$ is always non-idempotent and hence does not possess a bounded approximate identity. Since clearly $R$ is a Banach space with a Schauder basis, $R$ has BAP. By Theorem 4.1, the global dimension of every commutative Banach algebra $A$ whose radical is $R$ is strictly greater than one.

As a second example, let $R=\{f \in C[0,1]: f(0)=0\}$ with uniform norm, but convolution multiplication (see [6, Example 5.3]). This radical algebra is idempotent, but with no bounded approximate identity. If $A$ is a commutative Banach algebra and its radical is $R$, then $\operatorname{dg} A>1$.

Theorem 4.2. Let $A$ be a commutative Banach algebra, and let $R$ be the radical of $A$. Let I be a non-idempotent closed ideal in $R$, such that

(i) $\overline{R \cdot I}=I$;

(ii) I has $A P$;

(iii) $T(I) \subset I$ for each $T \in_{R} h(I, R)$.

Then $I$ is a closed ideal in $A$ and $I$ is not projective in $A-$ mod.

Proof. Using the condition (i) and the fact that $R$ is a closed ideal in $A$, it is easy to show that $I$ is an ideal in $A$ and that ${ }_{A} h\left(I, A_{+}\right)={ }_{R} h(I, R)$. The rest follows from Theorem 2.3.

Corollary 4.3. Let $A$ be a commutative Banach algebra, and let $R$ be the radical of $A$. Suppose that $R$ has $A P$, possesses an approximate identity and contains a nonidempotent, complemented closed ideal I such that $T(I) \subset I$ for each $T \in{ }_{R} h(I, R)$. Then $\operatorname{dg} A>1$.

Proof. Again, because of Helemskii's theorem we can assume that the spectrum of $A$ is finite. Since $R$ has AP and $I$ is a complemented closed subspace of $R, I$ has also AP. Since $R$ possesses an approximate identity, $\overline{R \cdot I}=I$. By Theorem 4.2, $I$ is a nonprojective closed ideal in $A$. Since $R$ is a closed subspace of finite codimension in $A, I$ has a Banach complement in $A$. By Lemma $2.1, \operatorname{dg} A>1$.

Now we can apply Theorem 4.1 and Corollary 4.3 to the study of cohomology groups of Banach algebras. Let $A$ be a Banach algebra, and let $U \in A$-mod- $A$. As in [13], we denote by $\mathcal{H}^{2}(A, U)$ the two-dimensional continuous Hochschild cohomology group of $A$ with coefficients in $U$. Recall that, for $X, Y \in A$-mod, the space $\mathfrak{B}(X, Y)$ can be regarded as a Banach $A$-bimodule by defining $(a \cdot \varphi)(x)=a \cdot \varphi(x)$ and 
$(\varphi \cdot a)(x)=\varphi(a \cdot x)(a \in A, \varphi \in \mathfrak{B}(X, Y), x \in X)$. It is known (see [13, Theorems III.5.4 and III.4.12]) that the following properties of $X$ are equivalent:

(i) $\mathrm{dh}_{A} X \leq 1$;

(ii) $\mathcal{H}^{2}(A, \mathfrak{B}(X, Y))=0$ for all $Y \in A$-mod.

So $\operatorname{dg} A \leq 1$ if and only if $\mathcal{H}^{2}(A, \mathfrak{B}(X, Y))=0$ for all $X, Y \in A$-mod.

Theorem 4.4. Let $R$ be a radical commutative Banach algebra with BAP. Let $R$ satisfy at least one of the following conditions:

(i) $R$ does not possess a bounded approximate identity;

(ii) $R$ contains a non-idempotent, complemented closed ideal $I$ such that $T(I) \subset I$ for each $T \in_{R} h(I, R)$.

Then for every commutative Banach algebra $A$ with radical $R$ there exists a Banach $A$-module $X$ such that $\mathcal{H}^{2}(A, X) \neq 0$.

For example, let $R$ be the Banach algebra $L^{1}(\omega)$, where $\omega$ is a radical weight function on $[0, \infty)$ (see Section 3). It is obvious that the Banach space $L^{1}(\omega)$ is isometrically isomorphic to the space $L^{1}(0, \infty)$ and hence has BAP. We recall (Theorem 3.3) that, if the (radical) algebra $R=L^{1}(\omega)$ possesses a bounded approximate identity, then the condition (iii) in Theorem 4.2 holds for each so-called standard ideal $I_{a}(a>0)$. Since always $\overline{\left(I_{a}\right)^{2}} \subset I_{2 a}$, all ideals $I_{a}(0<a<\infty)$ are not idempotent. These ideals are complemented subspaces in $R$ and hence have AP. Thus by combining Theorems 4.2 and 3.3 we obtain the following.

Theorem 4.5. Let $A$ be a commutative Banach algebra such that its radical is $L^{\prime}(\omega)$ (for example, $A=L^{1}(\omega)$, where $\omega$ is a radical weight function on $[0, \infty)$ ). Suppose that $L^{\prime}(\omega)$ possesses a bounded approximate identity. Then each standard ideal $I_{a}(0<a<\infty)$ of $L^{1}(\omega)$ is a non-projective closed ideal in $A$.

The following result is a consequence of Theorems 4.1, 3.3 and Corollary 4.3.

Theorem 4.6. Let $A$ be a commutative Banach algebra such that its radical is $L^{1}(\omega)$, where $\omega$ is a radical weight function on $[0, \infty)$. Then $\operatorname{dg} A>1$ and, as a consequence, there exists a Banach $A$-bimodule $X$ such that $\mathcal{H}^{2}(A, X) \neq 0$.

Now we are in a position to estimate the global dimension of any weighted convolution algebra. Let $\omega$ be any weight function on $[0, \infty)$, and set $\sigma=\lim _{t \rightarrow \infty} \omega(t)^{1 / t}$. Recall [3, Theorem 4.4] that $L^{\prime}(\omega)$ is a semi-simple (for $\sigma>0$ ) or radical (for $\sigma=0$ ) Banach algebra. Using Theorem 4.6 and Helemskii's theorem, we obtain the following.

Corollary 4.7. Let $\omega$ be any (measurable) weight function, and let $A=L^{\prime}(\omega)$. Then $\operatorname{dg} A>1$ and, as a consequence, there exists a Banach $A$-bimodule $X$ such that $\mathcal{H}^{2}(A, X) \neq 0$. 
We recall (see [13]) that the question of the triviality of two-dimensional cohomology groups of a Banach algebra $A$ with coefficients in Banach $A$-bimodules is closely connected with the question of the splitting of singular (admissible) extensions of this algebra. In particular, we obtain the next corollary from Theorem 4.6 and [13, Corollary I.1.11].

Corollary 4.8. Let $A$ be a commutative Banach algebra such that its radical is $L^{\prime}(\omega)$, where $\omega$ is a radical weight function on $[0, \infty)$. Then the Banach algebra $A$ has a singular extension that does not split.

\section{REFERENCES}

1. W. G. Bade and H. G. Dales, Norms and ideals in radical convolution algebras, J. Funct. Anal. 41 (1981), 77-109.

2. W. G. BADE, H. G. Dales and K. B. LaURSEN, Multipliers of radical Banach algebras of power series (Mem. Amer. Math. Soc. 303, 1984).

3. H. G. DALES, Convolution algebras on the real line, in Proceedings of Conference on Radical Banach Algebras and Automatic Continuity (Lecture Notes in Mathematics 975, SpringerVerlag, Berlin, 1983).

4. H. G. Dales and J. P. MCCluRE, Nonstandard ideals in radical convolution algebras on a half-line, Canad. J. Math. 39 (1987), 309-321.

5. M. Despić, F. Ghahramani and S. Grabiner, Weighted convolution algebras without bounded approximate identities, Math. Scand. 76 (1995), 257-272.

6. P. G. Dixon and G. A. Willis, Approximate identities in extensions of topologically nilpotent Banach algebras, Proc. Roy. Soc. Edinburgh 122A (1992), 45-52.

7. Y. Domar, Extensions of the Titchmarsh convolution theorem with applications in the theory of invariant subspaces, Proc. London Math. Soc. 46 (1983), 288-300.

8. P. ENFLo, A counterexample to the approximation problem in Banach spaces, Acta Math. 130 (1973), 309-317.

9. A. Grothendeck, Produits tensoriels topologiques et espaces nucleaires (Mem. Amer. Math. Soc. 16, 1955).

10. A. Ya. HelemsKiI, On the homological dimension of normed modules over Banach algebras, Mat. Sb. 81(123) (1970), 430-444 (Russian); English transl. in Math. USSR-Sb. 10 (1970), 399-411.

11. A. YA. Helemskil, Global dimension of a Banach function algebra is different from one, Funktsional. Anal. i Prilozhen. 6 (1972), 95-96 (Russian); English transl. in Functional Anal. Appl. 6 (1972), 166-168.

12. A. Ya. Helemskir, Smallest values assumed by the global homological dimension of Banach function algebras, Trudy Sem. Petrovsk. 3 (1978), 223-242 (Russian); English transl. in Amer. Math. Soc. Transl. 124 (1984), 75-96.

13. A. YA. HelemsKII, The homology of Banach and topological algebras (Moscow Univ. Press, 1986 (Russian); English transl.: Kluwer Academic Press, Dordrecht, 1989). 
14. A. YA. Helemskir, 31 problems of the homology of the algebras of analysis, in Linear and complex analysis problem book 3, Part I (V. P. Havin, N. K. Nikolski, eds., Lecture Notes in Math. 1573, Springer-Verlag, Berlin, 1994), 54-78.

15. E. Hille and R. S. Philips, Functional Analysis and Semi-groups (Colloquium Publication Series, 31, Amer. Math. Soc., Providence, R.I., 1957).

16. J. Lindenstrauss and L. TZAFriri, Classical Banach spaces I, Sequence spaces (Ergebnisse Math. 92, Springer-Verlag, New York, 1977).

17. Yu. V. Selivanov, Computing and estimating the global dimension in certain classes of Banach algebras, Math. Scand. 72 (1993), 85-98.

18. Yu. V. Selivanov, Projective ideals and estimating the global dimension of commutative Banach algebras, in Third International Conference on Algebra (Krasnoyarsk, 1993), 297-298 (Russian).

19. Yu. V. Selivanov, Projective Fréchet modules with the approximation property, Uspekhi Matem. Nauk 50:1 (1995), 209-210 (Russian); English transl. in Russian Math. Surveys. 50:1 (1995), 211-213.

20. YU. V. Selivanov, Homological characterizations of the approximation property for Banach spaces, Glasgow Math. J. 34 (1992), 229-239.

21. J. K. WANG, Multipliers of commutative Banach algebras, Pacific J. Math. 11 (1961), $1131-1149$.

UNIVERSITY OF MANITOBA

WINNIPEG, MANITOBA

CANADA R3T 2N2

Russian State University of Aviation Technology Petrovka 27, Moscow

Email address: ghahram@cc.umanitoba.ca 